\title{
THERMALLY STABLE HYBRID CAVITY LASER BASED ON SILICON NITRIDE GRATINGS
}

\author{
S. Iadanza ${ }^{1,2}$, A.P. Bakoz ${ }^{1,2}$, P.K.J. Singaravelu ${ }^{1,2}$, D. Panettieri ${ }^{1}$, S.A. Schulz ${ }^{1,2,3}$, G.C.R. \\ Devarapu $^{1,2}$, S. Guerber ${ }^{4}$, C. Baudot ${ }^{4}$, F. Boeuf ${ }^{4}$, S. Hegarty ${ }^{1,2}$, And L. O’FaOlain ${ }^{1,2,3}$ \\ ${ }^{1}$ Centre for Advanced Photonics and Process Analysis, Cork Institute of Technology, Cork, Ireland \\ ${ }^{2}$ Tyndall National Institute, Lee Maltings, Dyke Parade, Cork, Ireland \\ ${ }^{3}$ Scottish Universities Physics Alliance (SUPA), School of Physics and Astronomy, St Andrews, UK \\ ${ }^{4}$ STMicroelectronics, Crolles, France
}

In this paper we show experimental results of a thermally stable $\mathrm{Si}_{3} \mathrm{~N}_{4}$ external cavity ( $\mathrm{SiN} \mathrm{EC}$ ) laser with high power output and the lowest SiN EC laser threshold to our knowledge. The device consists in a 250 $\mu \mathrm{m}$ sized Reflective Semiconductor Optical Amplifier (RSOA) butt-coupled to a passive chip based on a series of $S_{3} N_{4}$ Bragg gratings acting as narrow reflectors. A threshold of $12 \mathrm{~mA}$ has been achieved, with a typical Side-Mode Suppression Ratio of $45 \mathrm{~dB}$ and measured power output higher than $3 \mathrm{~mW}$. Furthermore, we achieved a mode-hop free lasing regime in the range of $15 \mathrm{~mA}$ to $62 \mathrm{~mA}$ and wavelength thermal stability up to $80^{\circ} \mathrm{C}$. This solves the challenges related to cavity resonances thermal shift and shows the possibility for this device to be integrated in Dense WDM and heat intensive optical-interconnects technologies @ 2018 Optical Society of America

OCIS codes: (140.3490) Lasers, distributed feedback; (140.0140) Lasers and laser optics;(220.0220) Optical design and fabrication

http://dx.doi.org/10.1364/ao.XX.XXXXXX

\section{INTRODUCTION}

The ever-growing demand for higher data rates and bandwidth in fibre-optic communications and on-chip interconnections [1-4] has led to an increasing need for cheap, efficient and compact narrow linewidth semiconductor laser sources for applications in dense Wavelength Division Multiplexing (dWDM) technology, which offers broad bandwidth via the large number of channels available. In datacenter applications, Such interconnects are exposed to strong fluctuations in the operating temperature, making the quest for accurate wavelength control and stability fundamental to their success in WDM applications $[5,6]$. Furthermore, cost-effective and efficient single chip integration of photonics and optics can only be achieved through Complementary Metal-Oxide Semiconductor (CMOS) processing, in which high yield and fabrication repeatability are paramount. Although the Silicon-On-Insulator (SOI) platform possesses promising light propagation properties [7], the absence of efficient laser sources in silicon, due to its indirect band gap, is the key limiting factor for this platform. This issue is partially addressed by the integration of III-V semiconductors as gain medium for WDM lasers. Direct growth of III-V materials on Silicon platforms is extremely challenging, owing to lattice constant mismatches and compatibility issues, and hybrid [8] and heterogeneous [9] integration schemes have become popular. Our preferred configuration for the gain integration, completely compatible with wafer bonding, is the deployment of hybrid external cavity lasers (EC lasers), as demonstrated by A. J. Zilkie et al. [10], consisting of a commercially available Reflective Semiconductor Optical Amplifier (RSOA) chip butt-coupled to a passive Si-chip with a narrow-band reflector. This approach allows independent optimization and fabrication of passive and gain chips and external modulation of the laser. Currently, Coarse WDM optical links in datacenters are mostly based on DBR lasers and external silicon modulators [11].

Since Silicon has a high thermo-optic coefficient (TOC), it requires active cooling to maintain a sta- 
ble output wavelength [12], which is inefficient in terms of energy and cost, or else it requires the use of wide channel spacing, making WDM inefficient [13]. To deal with thermal drifts, integration of microring feedback controls [14], loop mirrors and MachZehnder Interferometers as frequency discriminators $[15,16]$ have been demonstrated. Yet, these solutions fail to address the thermal stability issue as thermal tuning is energy inefficient and silicon rings do not provide an athermal reference. However $\mathrm{Si}_{3} \mathrm{~N}_{4}$ is completely CMOS-compatible and has a five times lower TOC and lower free carrier absorption compared to $\mathrm{Si}$, offering excellent thermal stability for the laser operation. In this work, we demonstrate a hybrid, low threshold $\mathrm{Si}_{3} \mathrm{~N}_{4}$ laser system without the need for active tuning of any kind. with the system exhibiting a mode-hop free lasing regime over a wide range of injected current and wavelength thermal stability up to $80^{\circ} \mathrm{C}$. The $\mathrm{Si}_{3} \mathrm{~N}_{4}$ based hybrid laser reported here offers a very promising solution for dense WDM applications in datacenters and is ready for fabrication on the wafer scale.

\section{CONCEPT AND DESIGN}

The high thermal stability of the system has been achieved by our development of a hybrid external cavity laser, in which an RSOA gain chip is butt-coupled to a SiN Bragg grating which acts as the resonant mirror of the laser cavity. The schematic of the EC laser is shown in Figure 1a. The possibility to separately design and fabricate the gain and passive sections of the system has been exploited to allow each of the components to be independently optimized. Typically, such different components would have a low coupling efficiency due to mismatch of the waveguide modes. Here, we have designed the $\mathrm{SiN}$ waveguide to have a mode area closely matching that of the RSOA, creating a low loss interface between the sections, achieving $80 \%$ efficiency with $1 \mu \mathrm{m}$ tolerance (see Fig. $1 \mathrm{~b}$ ). The RSOA provides gain using quaternary quantum wells in AlInGaAs. The $\mathrm{Al}^{3+}$ ions present therein lead to a deepening of the potential well and impede carriers leakage at higher temperatures, improving higher temperature operation [17]. Due to the high gain material, the length of the gain chip used is as short as $250 \mu \mathrm{m}$, many times shorter than the competing solutions $[9,18]$, hence leading to very cost effective employment of III-V materials. The Bragg gratings are part of the actual $2 \mu \mathrm{m}$ wide $\mathrm{SiN}$ waveg- uides, greatly reducing the reflector footprint, thus unlocking high integration density and allowing a high channel count. The gratings were designed for a UV photolithography compatible approach that leads to volume manufacture and decrease in device costs. The Bragg gratings were designed to exploit the $2^{\text {nd }}$ order photonic band-gap, with a narrower stop-band with respect to the $1^{\text {st }}$ order band-gap, targeting a narrower FWHM of reflection peak, approximately $0.9 \mathrm{~nm}$. During operation, carriers injected recombine in the quantum wells of the RSOA emitting light, which is then coupled to the SiN waveguide and propagates towards the mirror through the waveguide. The light component that is off resonance with the gratings is transmitted to the output facet of the waveguide while the on-resonance component is partially reflected back to the RSOA. A wavelength-selective feedback is thus generated, with a linewidth on the order of 0.9 $\mathrm{nm}$, forming a laser cavity between the gratings and the reflective facet of the RSOA (as shown in Fig. 1c). The emitted laser wavelength corresponds to the longitudinal cavity mode that lies in the resonant response of the gratings. The parameters of the laser can be calculated from the combination of the gain curve and the resonance characteristics of the laser cavity. The longitudinal mode spacing of a composite cavity, $\Delta v$, is defined as the speed of light divided by twice the sum of the optical path lengths of each element in the cavity. The corresponding wavelength spacing, $\Delta \lambda$, is:

$$
\Delta \lambda=\frac{\lambda^{2}}{2\left(l_{\text {gain }} n_{\text {gain }}+\left(n_{\text {passive }}\right)\left(l_{\text {passive }}+l_{\text {grating }}\right)\right)}
$$

Where $l_{\text {gain }}$ and $n_{\text {gain }}$ are the length of the gain chip and its group refractive index respectively. $l_{\text {passive }}$ and $l_{\text {grating }}$ are the lengths of the waveguide and the effective length of the gratings respectively, with $n_{\text {passive }}$ being the group refractive index of the passive chip. The lasing threshold is reached when the laser optical gain is perfectly balanced by the total loss experienced by light in one round trip of the laser optical cavity. In a steady-state operation, the threshold condition $\mathrm{g}$ can be defined as:

$$
g=\alpha_{i}+\left(\frac{1}{l_{\text {gain }}}\right) \ln \left(\frac{1}{\kappa^{2}\left(r_{R S O A}\right)\left(r_{\text {grating }}\right)}\right)
$$

where $\alpha_{i}$ represent the optical loss coefficient, $r_{\text {grating }}$ and $r_{R S O A}$ are the grating and RSOA reflectivities and $\kappa$ is the coupling coefficient between the 
RSOA and the SiN waveguide of the passive chip and is determined from Fig. 1b).

\section{METHODS AND REPEATABILITY STUDY}

The gain chip consisted of the RSOA mounted on an AlN ceramic tile and glued to an Al holder, to facilitate the alignment. The chip was wire-bonded to a current and temperature controller. A rototranslational stage was used to align the gain chip to the passive device, mounted on the sample-holder, similar to the setup described in [10] in edge free space coupling configuration. The RSOA consists of a $250 \mu \mathrm{m}$ long AlInGaAs ridge waveguide amplifier, with the back facet HR coated to exhibit reflectivity $\mathrm{R}$ higher than $90 \%$ and the front end AR coated to suppress back-reflections in the amplification loop. The RSOA showed still high gain at high temperatures up to $80^{\circ} \mathrm{C}$. The gain optical emission was characterized as a function of driving current and temperature and showed modulated spectra with a fringe spacing of $1.34 \mathrm{~nm}$, related to the Fabry-Perot interference generated by the facets of the RSOA. The SiN device was fabricated on the $300 \mathrm{~mm}$ R\&D silicon photonic platform of STMicroelectronics Crolles, France [19, 20]. Fabrication starts with a $1.4 \mu \mathrm{m}$ thick buried silicon dioxide layer growth on a silicon bulk wafer. A 600 $\mathrm{nm}$ SiN layer was then deposited at low temperature PECVD. SiN patterning is performed with optical DUV lithography followed by dry etching. An encapsulation layer of a $1.5 \mu \mathrm{m}$ thick silicon dioxide layer was used to complete the process. The SiN passive chip has been AR-coated with a layer of $282 \mathrm{~nm}$ thick $\mathrm{MgO}$, to suppress Fabry-Perot modulations related to facet-to-facet reflections of the waveguides.

The dimensions of the SiN gratings are crucial parameters in determining the lasing wavelength of the proposed hybrid EC laser. The spectral responses of the reflectors depend on the periodicity and corrugation of the gratings and on the width and height of the waveguides. A good reproducibility of the $\mathrm{SiN}$ reflectors and a high fabrication yield are crucial for high volumes manufacturing. In order to verify repeatability and high yield, the inter-die variations of the $300 \mathrm{~mm}$ wide SiN on SOI wafer have been studied in this work. 20 chips in total, 5 per quadrant, were cleaved to be characterized optically. On those chips, 4 different devices with the same grating period of $510 \mathrm{~nm}$, and different waveguide widths of 0.9 $\mu \mathrm{m}, 1.1 \mu \mathrm{m}, 1.3 \mu \mathrm{m}$ and $1.5 \mu \mathrm{m}$ respectively, were selected for the acquisition of the optical spectra on the

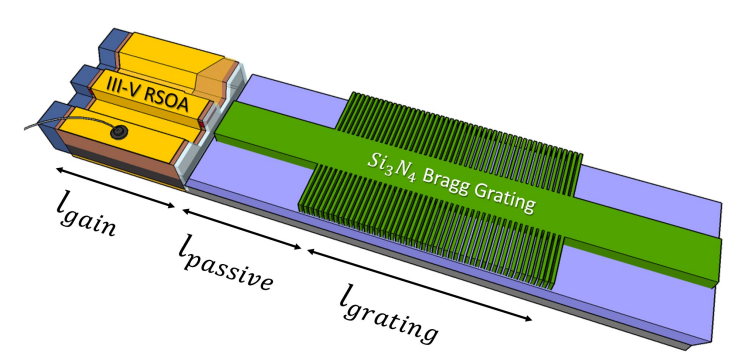

(a)

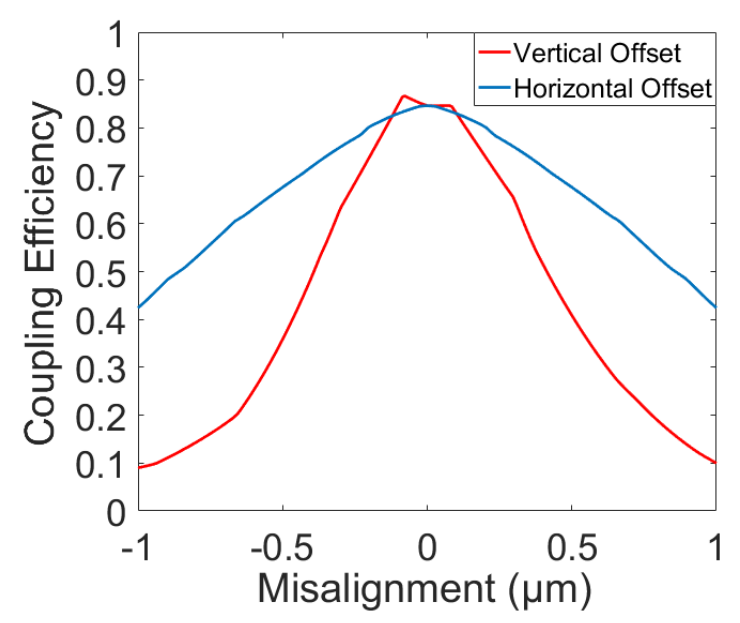

(b)

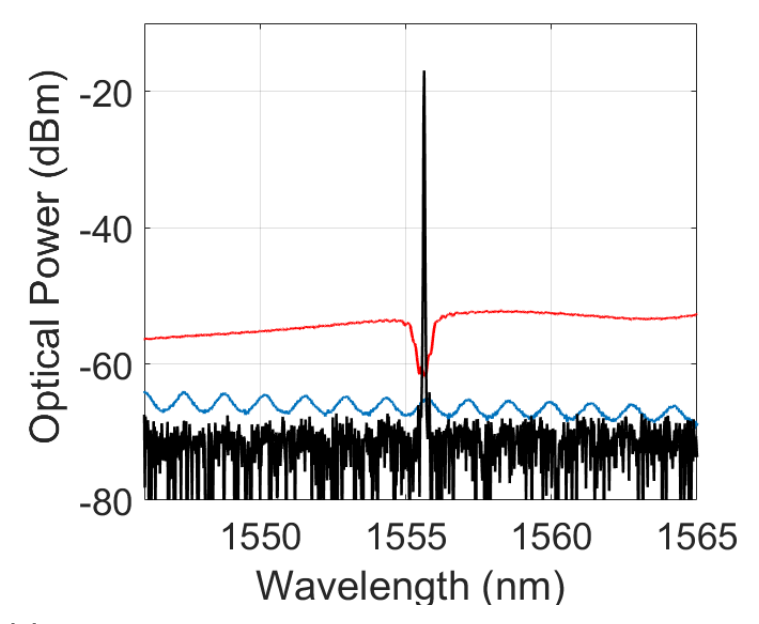

(c)

Fig. 1. (a) Schematics of the hybrid EC Lase based on the butt-coupled RSOA and SiN mirror. The reflector comprise a $\mathrm{SiN}$ waveguide with a Bragg grating etched on it, on a Silicon Dioxide substrate and cladded in a low-index flowable oxide. (b) Simulated Coupling Efficiency vs. Misalignment between the SiN waveguide and the butt-coupled RSOA. (c) Power vs. Wavelength plot, showing the spectral overlap of the gain ripple (blue curve), the SiN Bragg mirror (red curve) and the total cavity laser spectrum (black curve). 


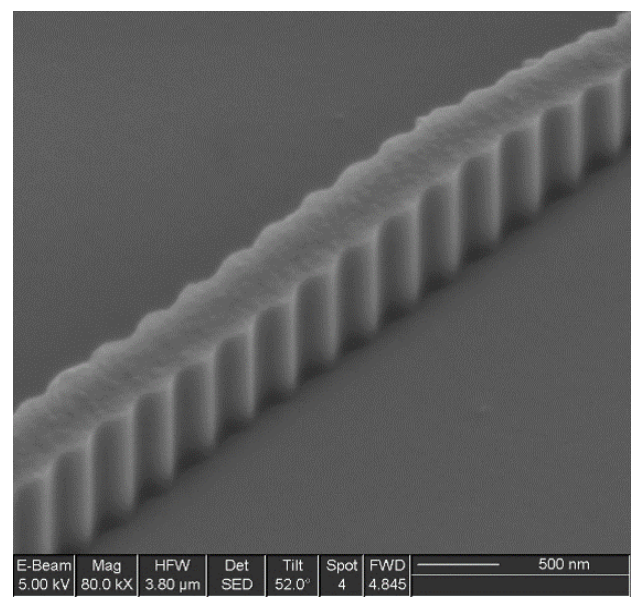

(a)

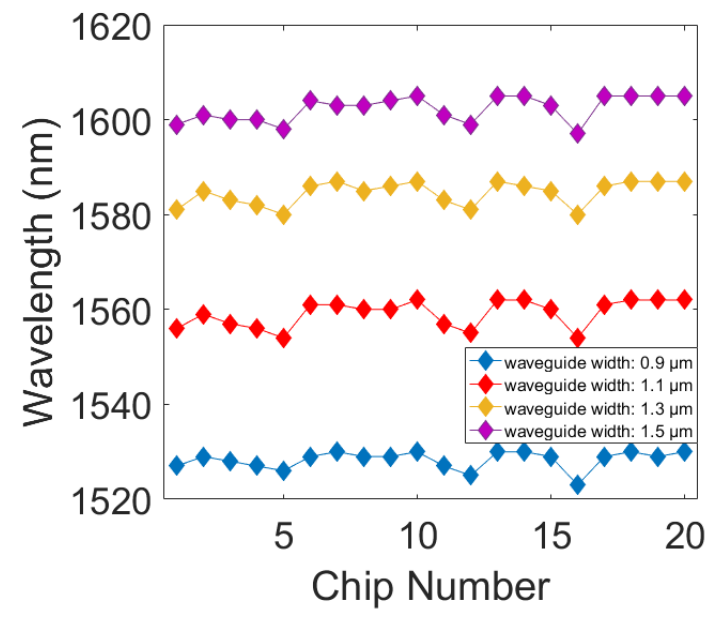

(b)

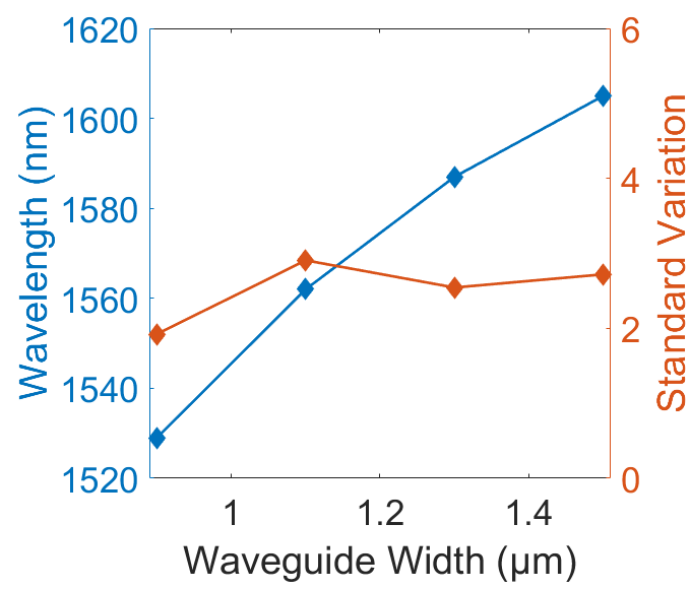

(c)

Fig. 2. (a) Tilted SEM view of the $600 \mathrm{~nm}$ thick patterned silicon nitride before encapsulation. (b) Plot of the measured reflection peaks central wavelengths of the 4 devices on all the 20 chips. Waveguide widths are $0.9 \mu \mathrm{m}$ (blue curve), $1.1 \mu \mathrm{m}$ (red curve), $1.3 \mu \mathrm{m}$ (yellow curve) and $1.5 \mu \mathrm{m}$ (purple curve). (c) Blue axis shows the most frequent resonant wavelengths over all the 20 chips at each waveguide width and the red axis shows the resonant wavelengths standard deviations over all the chips at each waveguide width. setup described in [10], and the Bragg reflection peak central wavelengths have been plotted for every device. In Fig. $2 b$ is shown that all the 4 devices on each chip follow the same behavior, shifted in wavelength depending on the waveguide width. The standard deviation of the resonance wavelengths is within the range of 2-3 nm and the average shift is $\pm 7 \mathrm{~nm}$ over all the 4 waveguide widths. Hence, these reflectors can be mass produced on the on wafer scale through the photolithographic process described earlier, with no concerns on the fabrication yield.

\section{RESULTS AND DISCUSSION}

The thermal stability achieved with our hybrid EC laser was fundamentally dependent on the thermal behavior of the material of the passive chip. The modes of the laser cavity depend on the product of the refractive index and the length of the passive chip (as defined in the previous section). A temperature change on the material would lead to a change of its refractive index by thermo-optic effect, hence shifting in wavelength the reflection peak of the gratings and also changing the effective optical length of the laser cavity, affecting the lasing wavelength and the laser thermal behavior. To verify the thermal stability of the passive chip, we proceeded with calculating the thermo-optic coefficient of the SiN. This was carried out by experimentally measuring the wavelength shift of the gratings reflection peak with temperature. A change in the device temperature leads to a change in both group and effective refractive indexes of the material, thus changing the Bragg condition of the grating and shifting the reflector peak wavelength - the thermo-optic effect. For this thermal study, initially reflection and transmission spectra of the gratings on the SiN passive chip (prior to AR coating) have been acquired at room temperature and exhibited Fabry-Perot fringes superimposed on the Bragg reflection peak. This fringe spacing $\Delta \lambda_{\text {fringes }}$ is related to the optical properties of the device and its length by:

$$
\Delta \lambda_{\text {fringes }}=\frac{\lambda^{2}}{2 n_{g} L_{\text {chip }}}
$$

A $\Delta \lambda_{\text {fringes }}$ of $0.097 \mathrm{~nm}$ has been measured at $20^{\circ} \mathrm{C}$. This value corresponds to a group index, $n_{g}$, of the cladded SiN waveguide of 2.03, with the passive chip length being $6.1 \mathrm{~mm}$, with the grating being $1.57 \mathrm{~mm}$ long. This value is in good agreement with the simulated group index, $n_{g}^{\text {sim }}=2.01$ of the structure, with 
over $92 \%$ of the fundamental TE mode confined in the $\mathrm{SiN}$ waveguide. In the next step, thermal characterization of the SiN material has been carried out by mounting it on a thermo-electric stage implemented in the setup for the heating and cooling. Optical spectra of the SiN Bragg grating have been measured at temperatures starting from $20^{\circ} \mathrm{C}$, up to $80^{\circ} \mathrm{C}$ with a $5^{\circ} \mathrm{C}$ step. In Fig. 3, the central wavelength of the reflection peaks has been plotted against temperature. It is remarkable to notice that the Bragg reflector central wavelength is shifted by a mere $0.744 \mathrm{~nm}$, from $\lambda_{0}=1556.048 \mathrm{~nm}$ at $20^{\circ} \mathrm{C}$ to $\lambda_{1}=1556.792 \mathrm{~nm}$ at $80^{\circ} \mathrm{C}$, resulting in the measured thermal sensitivity of the SiN Bragg reflector of $12.4 \mathrm{pm} /{ }^{\circ} \mathrm{C}$.

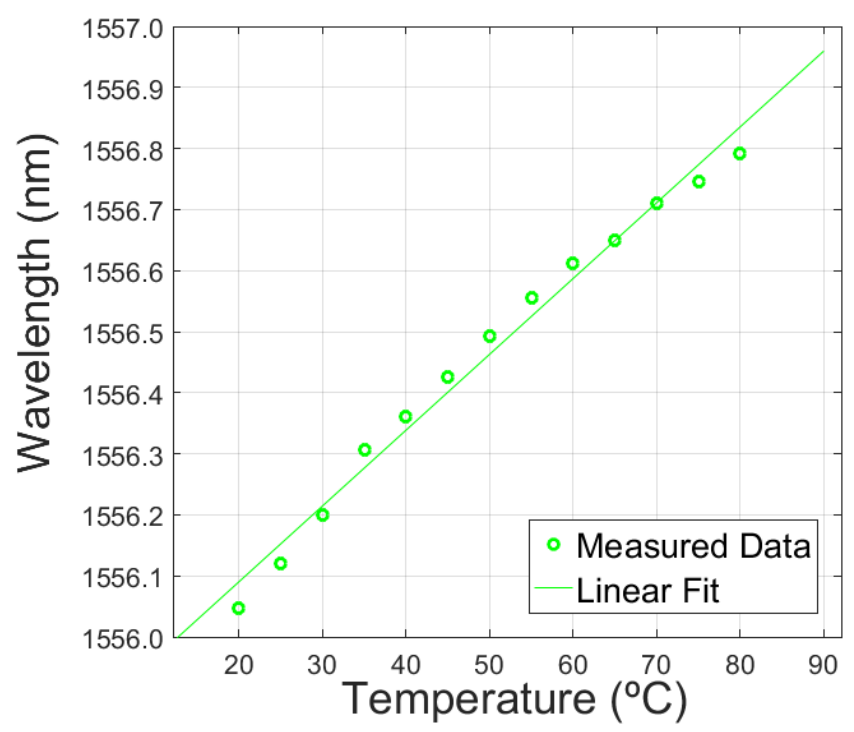

Fig. 3. Plot of the central wavelength of the Bragg grating reflection peak as a function of operating temperature. The device displays a constant shift of $12.4 \mathrm{pm} /{ }^{\circ} \mathrm{C}$

From this measured thermal sensitivity, the corresponding thermo-optic coefficient (TOC) can be determined through the following relation [21, 22]:

$$
\text { TOC }=\frac{1}{\Delta T} \frac{\left(\lambda_{1}-\lambda_{0}\right)}{\lambda_{1}} n_{g}^{\text {room }}=1.62 * 10^{-5} \frac{\text { RIU }}{{ }^{\circ} \mathrm{C}}
$$

We attribute the above thermal behaviour of the device to be purely caused by the TOC of SiN, since the high confinement of the mode in the $\mathrm{SiN}$ region and the very low TOC of the substrate and cladding being $9.5 * 10^{-6} \mathrm{RIU} /{ }^{\circ} \mathrm{C}[21]$. Our experimental value of the SiN TOC is in good agreement with [23]. This measured value is lower than the TOC of $2.5 * 10^{-5}$
$\mathrm{RIU} /{ }^{\circ} \mathrm{C}$ for N-rich SiN reported in [24]. The mismatch is probably due to the difference in stoichiometry of the SiN layers of both materials.

To demonstrate the athermal operation of our proposed hybrid laser, the characteristics of several $\mathrm{SiN}$ gratings that were butt-coupled to the quaternary RSOA to form the EC lasers have been measured with increasing driving currents at room temperature $\left(20^{\circ} \mathrm{C}\right)$, without active cooling for drive currents up to $100 \mathrm{~mA}$, with a $1 \mathrm{~mA}$ current steps. Lasing spectra were acquired at each current step.

In Fig. 4a, the time-averaged optical spectrum of one of such EC Laser was plotted in a false color map as a function of the drive current. Notice the single mode lasing regime free of mode-hopping achieved from $15 \mathrm{~mA}$ to $62 \mathrm{~mA}$ of drive current. This single longitudinal mode lasing was attained by matching the longitudinal mode of the total laser cavity with the Bragg reflection peak (as seen in Fig. 1c). Moreover, it can be noticed from Fig. 4a that our EC laser has a threshold current as low as $10 \mathrm{~mA}$ and has output power up to $3 \mathrm{~mW}$ as shown in Fig. 4c. The emitted output power was measured by collimating the outcoming beam with a lens on to a power meter sensor head.

All of the EC lasers that have been characterized have such low threshold currents in the range of 10 $\mathrm{mA}$ and $20 \mathrm{~mA}$, with the lowest values of $10 \mathrm{~mA}$ corresponding to the gratings with the highest reflectivity (R 78\%). The output power was measured to be in the range of several $\mathrm{mW}$ for all of the characterized devices.

Over the full mode-hop free lasing regime shown in Fig. 4a, a side-mode suppression rate (SMSR) higher than $40 \mathrm{~dB}$ was measured, with a maximum of $49 \mathrm{~dB}$ as shown in Fig. $4 \mathrm{~d}$. Linewidth of the single mode laser was measured with a tunable laser source through a delayed heterodyne measurement, and was found to be lower than $3 \mathrm{MHz}$ over the complete operating regime.

When the driving current was increased beyond $65 \mathrm{~mA}$, the increased gain of the active chip led to lasing of the side modes near the centre wavelength of the reflector. This can be seen Fig. 4a, where the time averaged optical spectra shows two main lasing modes, with additional, weaker sidebands starting to appear, as a result of the averaging of time-varying random transitions occurring due to the competition of the two longitudinal modes.

After the above characterization of the devices at 


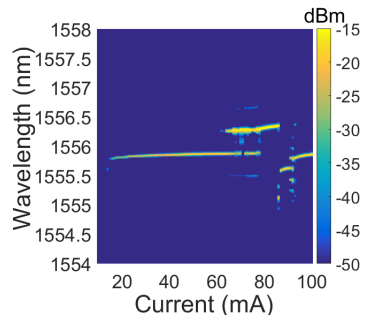

(a)

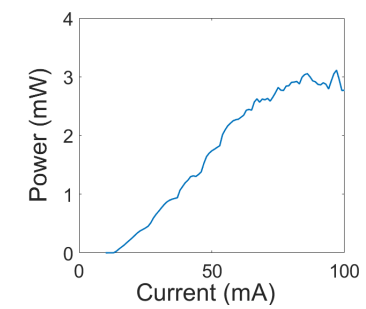

(c)

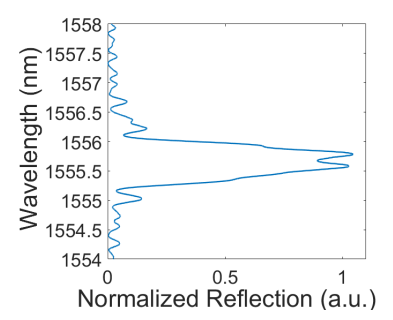

(b)

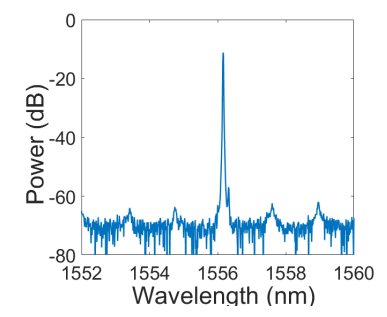

(d)

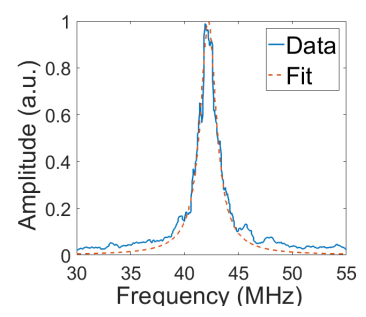

(e)

Fig. 4. Laser characterization. (a) False colour plot of the optical spectrum of the laser, averaged in time, with the increasing driving current. (b) Normalized reflection spectrum of the Bragg gratings, with the wavelength axis matched to that on the left, (c) L-I curve of the EC laser. (d) Time-averaged optical spectrum of the EC laser at room temperature and $50 \mathrm{~mA}$ of driving current. The laser line shows $49 \mathrm{~dB}$ of SMSR. (e) Linewidth of the hybrid EC laser and fit.

room temperature, the characteristics of multiple EC Laser devices were measured as a function of temperature by mounting both the RSOA and SiN gratings in butt-coupled fashion on two independent thermoelectric controlled plates, fixing the RSOA in place with conductive wax. The EC Laser spectra were collected with increasing driving current at each temperature, from $20^{\circ} \mathrm{C}$ to $80^{\circ} \mathrm{C}$. Fig. 5 shows the single mode lasing wavelength plotted against the temperature for a fixed driving current of $50 \mathrm{~mA}$. As the temperature of operation is increased, no red-shift of the lasing wavelength was observed, as opposed
$6 \mathrm{~nm}$ increase of the lasing wavelength that is associated with the traditional InP DFB for the same change in temperature (JDS CFQ935 [25]). Further on, the hybrid EC laser showed over $40 \mathrm{~dB}$ of SMSR and output power in the 1 to $3 \mathrm{~mW}$ range in the entire temperature interval considered.

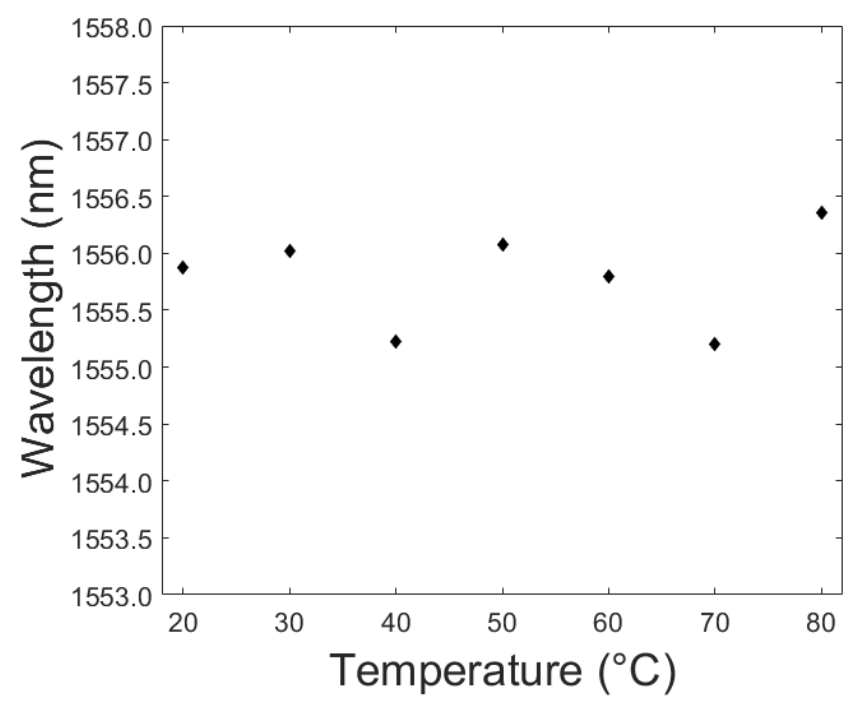

Fig. 5. Plot of the single mode lasing wavelength against temperature with the controlled driving current of $50 \mathrm{~mA}$

The lasing wavelength of our EC laser is determined by the resonance wavelength of our narrowband Bragg grating reflector. The use of a low TOC material, here $\mathrm{SiN}$, for the grating provides a negligible thermal shift of the Bragg reflector resonant wavelength and hence the lasing wavelength. This enhanced thermal stability of the lasing wavelength of the device over a temperature range of operation of $60^{\circ} \mathrm{C}$, completely eliminates the need of active cooling of the laser normally employed in Si-based DFBs.

\section{CONCLUSION}

In conclusion, we demonstrated a low threshold hybrid external-cavity laser based on a SiN reflector coupled to a RSOA. The laser is single mode and mode-hop free over a driving current range of 49 $\mathrm{mA}$, from $13 \mathrm{~mA}$ to $62 \mathrm{~mA}$, with a power output on the order of $\mathrm{mWs}$ over the total range.

We envisage that further improvement of the AR coatings of the SiN chip will lead to the widening of the mode-hop free operation regime to even higher driving currents. The use of narrower reflection peaks could lead to more stable operation and higher 
side mode suppression ration. However, reducing the contrast would lead to an increase of the passive chip length reducing the FSR of the laser cavity modes, cancelling out the benefits. For this reason, we propose the replacement of the gratings with $\mathrm{SiN}$ resonant mirrors such as $1 \mathrm{D}$ and 2D photonic crystals cavities [26], whose high Q-Factors and incredibly small footprints, even compared to gratings, would lead to a finer lasing wavelength control and wider mode-hop free regime.

Our hybrid EC Laser achieved a lasing wavelength stability of $0.16 \mathrm{~nm}$ in the total temperature range of operation $20^{\circ} \mathrm{C}-80^{\circ} \mathrm{C}$ with a $\mathrm{mW}$ range output power, compatible with WDM standards with $200 \mathrm{GHz}$ and $400 \mathrm{GHz}$ channel spacings. This paves the way for future employment of silicon nitride chips with a series of gratings or photonic crystal cavities resonant at different densely spaced wavelengths coupled to arrays of commercialized RSOAs, generating a dense network of compact transmitters, without the need of any active temperature control, thus greatly reducing the related power consumption and cost and making these lasers an excellent prospect for low cost Dense WDM applications in datacenters.

\section{FUNDING INFORMATION}

This work was supported by:

Science Foundation Ireland under Grant SFI12/RC/2276, and Grant 16/ERCS/3838

Engineering and Physical Sciences Research Council (EPSRC) (doctoral grant EP/L505079/1 and equipment grant EP/L017008/1);

European Union Horizon H2020 Programme (H2020-ICT27-2015, COSMICC nr. 688516) European Research Council (ERC) (Starting Grant 337508);

\section{REFERENCES}

1. R. Soref, "The Past, Present, and Future of Silicon Photonics," IEEE J. Sel. Top. Quantum Electron. 12, 1678-1687 (2006).

2. B. Jalali and S. Fathpour, "Silicon photonics," J. Light. Technol. 24, 4600-4615 (2006).

3. Krishnamoorthy and A. V., "Computer systems based on silicon photonic interconnects," in "Proceedings of the IEEE," , vol. 97 (2009), vol. 97, pp. 1337-1361.

4. Y. A. Vlasov, "Silicon cmos-integrated nano-photonics for computer and data communications beyond 100g," IEEE Commun. Mag. 50, s67-s72 (2012).

5. A. A. Liles, K. Debnath, and L. O'Faolain, "Lithographic wavelength control of an external cavity laser with a silicon photonic crystal cavitybased resonant reflector," Opt. Lett. 41, 894-897 (2016).

6. X. Zheng, S. Member, S. Lin, Y. Luo, J. Yao, G. Li, S. S. Djordjevic, J.-H. Lee, H. D. Thacker, I. Shubin, K. Raj, and J. E. Cunningham, "Efficient WDM Laser Sources Towards Terabyte / s Silicon Photonic Interconnects," J. Light. Technol. 31, 4142-4154 (2013).
7. L. Chen, C. R. Doerr, P. Dong, and Y. kai Chen, "Monolithic silicon chip with 10 modulator channels at 25 gbps and 100-ghz spacing," Opt. Express 19, B946-B951 (2011).

8. F. E. Doany, R. Budd, L. Schares, T. Huynh, M. Wood, D. Kuchta, N. Dupuis, C. Schow, B. Lee, M. Moehrle, A. Sigmund, W. Rehbein, T. Y. Liow, L. W. Luo, and G. Q. Lo, "A four-channel silicon photonic carrier with flip-chip integrated semiconductor optical amplifier (soa) array providing >10-db gain," in "2016 IEEE 66th Electronic Components and Technology Conference (ECTC)," (2016), pp. 1061-1068.

9. G.-H. Duan, C. Jany, A. Le Liepvre, A. Accard, M. Lamponi, D. Make, P. Kaspar, G. Levaufre, N. Girard, F. Lelarge, J.-M. Fedeli, S. Messaoudene, D. Bordel, and S. Olivier, "Hybrid III-V on silicon lasers for photonic integrated circuits on silicon," IEEE J. Sel. Top. Quantum Electron. 20, 158-170 (2014).

10. A. J. Zilkie, P. Seddighian, B. J. Bijlani, W. Qian, D. C. Lee, S. Fathololoumi, J. Fong, R. Shafiiha, D. Feng, B. J. Luff, X. Zheng, J. E. Cunningham, a. V. Krishnamoorthy, and M. Asghari, "Powerefficient III-V/silicon external cavity DBR lasers." Opt. express 20, 23456-62 (2012).

11. G. Yoffe, T. Nguyen, J. Heanue, and B. Pezeshki, "Efficient compact tunable laser for access networks using silicon ring resonators," in "OFC/NFOEC," (2012), pp. 1-3.

12. Optomark, "DWDM multiplexers," http://www.optomark.eu/products/ cwdm-dwdm/dwdm-multiplexers.html.

13. FibeReality, "100G Transceivers Below $\$ 3 /$ Gig by 2017?" http:// fibereality.com/blog/100g-transceivers-below-3gig-by-2017/.

14. J. H. Lee, D. Y. Lee, I. Shubin, J. Bovington, S. S. Djordjevic, S. Lin, Y. Luo, J. Yao, J. E. Cunningham, K. Raj, A. V. Krishnamoorthy, and $X$. Zheng, "III-V/Si Hybrid Laser Stabilization Using Micro-Ring Feedback Control," IEEE Photonics J. 8, 1-7 (2016).

15. C. Wang, X. Li, H. Jin, H. Yu, J. Yang, and X. Jiang, "Silicon reflectors for external cavity lasers based on ring resonators," Opt. Commun. 383, 453-459 (2017).

16. Y. Fan, J. P. Epping, R. M. Oldenbeuving, C. G. H. Roeloffzen, M. Hoekman, R. Dekker, R. G. Heideman, P. J. M. van der Slot, and K.-J. Boller, "Optically Integrated InP-Si3N4 Hybrid Laser," IEEE Photonics J. 8, 1-11 (2016).

17. E. Murphy, C. Michie, H. White, W. Johnstone, A. E. Kelly, and I. Andonovic, "High temperature wavelength division network for avionic applications," J. Light. Technol. 31, 3006-3013 (2013).

18. M. J. R. Heck, J. F. Bauters, M. L. Davenport, J. K. Doylend, S. Jain, G. Kurczveil, S. Srinivasan, Y. Tang, and J. E. Bowers, "Hybrid Silicon Photonic Integrated Circuit Technology," IEEE J. Sel. Top. Quantum Electron. 19, 6100117-6100117 (2013).

19. C. Baudot, M. Douix, S. Guerber, S. Crémer, N. Vulliet, J. Planchot, R. Blanc, L. Babaud, C. Alonso-Ramos, D. Benedikovich, D. PérezGalacho, S. Messaoudène, S. Kerdiles, P. Acosta-Alba, C. EuvrardColnat, E. Cassan, D. Marris-Morini, L. Vivien, and F. Boeuf, "Developments in $300 \mathrm{~mm}$ silicon photonics using traditional CMOS fabrication methods and materials," in "IEEE International Electron Devices Meeting," (2017), pp. 34.3.1-34.3.4.

20. S. Guerber, C. Alonso-Ramos, D. Perez-Galacho, X. Le Roux, N. Vulliet, S. Cremer, D. Marris-Morini, F. Boeuf, L. Vivien, and C. Baudot, "Design and integration of an O-band silicon nitride AWG for CWDM applications," in "14th International Conference on Group IV Photonics, GFP 2017," (2017), pp. 133-134.

21. A. Arbabi and L. L. Goddard, "Measurements of the refractive indices and thermo-optic coefficients of $\mathrm{Si}_{3} \mathrm{~N}_{4}$ and $\mathrm{SiO}_{x}$ using microring resonances," Opt. Lett. 38, 3878 (2013).

22. Z. Yu and S. Fan, "Extraordinarily high spectral sensitivity in refractive index sensors using multiple optical modes," Opt. Express 19, 1002910040 (2011).

23. K. Wörhoff, R. G. Heideman, A. Leinse, and M. Hoekman, "TriPleX: A versatile dielectric photonic platform," Adv. Opt. Technol. 4, 189-207 (2015).

24. T. Domínguez Bucio, A. Z. Khokhar, G. Z. Mashanovich, and F. Y. Gardes, "Athermal silicon nitride angled MMI wavelength division (de)multiplexers for the near-infrared," Opt. Express 25, 27310-27320 
(2017).

25. JDSUniphase, "50 mW $1550 \mathrm{~nm}$ CW DFB Lasers with PM Fiber for WDM Applications," http://www.lightwavestore.com/product_ datasheet/OSC-LDPM-L-110C_pdf1.pdf.

26. K. Debnath, L. O'Faolain, F. Y. Gardes, A. G. Steffan, G. T. Reed, and T. F. Krauss, "Cascaded modulator architecture for WDM applications." Opt. express 20, 27420-8 (2012). 